\title{
La périodisation en linguistique : problèmes théoriques et méthodologiques
}

\author{
Bernard Combettes \\ Université Nancy-2, ATILF \\ Christiane Marchello-Nizia \\ ENS-LSH, ICAR
}

Le changement linguistique est habituellement considéré comme un mouvement continu, même si certaines époques peuvent sembler plus ou moins stables que d'autres. La variation synchronique, qui est au fondement du changement et rend possible l'évolution (cf. la notion de variantes "qui réussissent" pour décrire les modifications de structures), est elle aussi toujours présente dans les langues. Dès lors la reconnaissance de "périodes" pose forcément problème dans la mesure où il s'agit de déterminer des ruptures dans ce qui apparaît comme un continuum.

Il y a là toutefois, dans la volonté souvent affirmée de reconnaître des étapes successives dans une histoire, une tradition constante dans l'analyse linguistique, et qui concerne les langues des familles les plus diverses, pour autant que leur histoire peut être documentée.

La périodisation correspond ainsi à un point de vue sur la diachronie, point de vue reposant sur des présupposés qui sont loin d'être toujours explicités. On rappellera par exemple l'influence qu'ont pu avoir, dans la périodisation du français, les modèles offerts par l'histoire littéraire ou par l'histoire de la civilisation (la reconnaissance des périodes du français du XVI ${ }^{\text {ème }}$ siècle ou de langue classique est explicitement justifiée, chez des auteurs comme Littré ou comme Nyrop, par l'existence des "époques littéraires" ou, plus largement, des "époques culturelles" correspondantes). On remarquera également qu'une périodisation n'a rien d'immuable : la notion de moyen français est construite par Darmesteter au début du $X X X^{\text {ème }}$ siècle, et ce n'est qu'assez récemment qu'émerge celle de français préclassique. De plus, la délimitation d'une période donnée ne fait pas toujours l'unanimité (on citera le cas du "moyen français", dont le terminus ad quem varie notablement selon les descriptions ; cf. Smith). Ce "figement" en segments temporels d'une réalité caractérisée par la continuité mérite une réflexion, tant du point de vue théorique que du point de vue méthodologique.

On doit ainsi se demander si la notion de 'périodes' est étrangère au système de la langue, "imposée" en quelque sorte de l'extérieur, et doit donc être exclue de l'analyse et de la réflexion linguistiques, ou bien si elle repose sur des caractères spécifiques de l'évolution des langues et relève alors de plein droit de l'étude du langage.

Une première constatation tendrait à valider l'idée qu'il existe des 'périodes' dans l'évolution des langues. Les diverses parties et sous-parties du système linguistique n'évoluent pas toutes de la même manière, à la même vitesse ; des tendances générales peuvent sans doute être identifiées, mais leur application dans le champ de la syntaxe, par exemple, ne s'opère pas obligatoirement en même temps que dans celui de la morphologie ou de la phonologie. Le lexique, quant à lui, constitue un domaine particulier dont les relations avec les autres niveaux ne peuvent être que très indirectes. Ainsi peut-on considérer, dans le cas du français, que le $\mathrm{XVI}^{\mathrm{ème}}$ siècle se caractérise par un renouvellement évident du lexique (renouvellement dû en grande partie à des causes externes, telles que le développement de l'humanisme ou des contacts avec l'Italie) et que cette évolution justifie l'identification d'une borne initiale qui limiterait le moyen français à la fin du $\mathrm{XV}^{\text {ème }}$ s. Il n'en va cependant pas de même pour ce qui est de la syntaxe ou de la morphologie, que des changements n'affecteront que vers le milieu du siècle, ce qui semble justifier un terminus a quo pour le français préclassique. On est là en présence d'un "décalage" typique, qui pourrait d'ailleurs être également observé dans le cas d'autres périodes si l'on disposait 
d'études détaillées sur la chronologie de l'évolution du lexique, et dont la prise en compte devrait pour le moins conduire à des distinctions plus fines que celles qui sont ordinairement pratiquées (on pense par exemple à des notions comme celle de "français classique" ou de "français moderne").

Cette non coïncidence entre les changements des différents niveaux peut d'ailleurs être retrouvée à l'intérieur même de chacun d'entre eux ; pour prendre l'exemple le plus richement documenté sans doute, celui de la syntaxe, il est facile de constater que la détermination nominale, l'ordre des mots, la construction des verbes, sont loin de marcher d'un même pas. S'il est possible de considérer qu'une grande tendance comme celle qui généralise l'ordre déterminé + déterminant et qui crée des hiérarchisations là où le latin présentait davantage des structures "plates", proches de la parataxe, sous-tend les modifications qui surviennent dans les divers sous-domaines de la structuration de la phrase, force est de constater que ce mouvement se réalise plus rapidement et plus complètement dans certaines parties de la syntaxe que dans d'autres (on pourra ainsi comparer la non expression du sujet et celle de l'article, la place des compléments essentiels et celle de l'épithète, etc.). En est-on réduit à ne prendre en considération que l'évolution de tel ou tel phénomène, avec les ruptures chronologiques qui le concernent, et à abandonner l'idée de regrouper de façon homogène la diversité des changements particuliers?

Dans ce cas, la seule 'périodisation' possible semblerait reposer sur des facteurs externes au système tels que ceux qu'a développés l'histoire 'externe' des langues (cf. Brunot, Chaurand, Rey et Siouffi, entre autres), spécialement dans une perspective, aujourd'hui bien représentée et riche de résultats, qui prend appui sur des critères sociolinguistiques (cf. Ayres-Bennett, Lodge, Lusignan, Gadet) : attitudes face à la langue (normativité), politique linguistique, phénomènes de 'modes', constitution d'un 'standard' (cf. Lodge), langues de groupe, emprunts, périodes de 'latinisation' (ou d'italianisation ou d'anglicisation), influence des usages littéraire ou technique, langues sexuées, phénomènes de langues en contact, etc.

Mais même si les avancées obtenues depuis deux décennies dans cette perspective ont mis en évidence l'importance de ces phénomènes, il s'agit toujours de pratiques variationnistes historicisables, ponctuelles et datables, mais qui ne constituent pas un processus continu avec des étapes. Ils nécessitent cependant que l'on repense l'articulation de ces faits de variation avec l'évolution des langues : dans quelle mesure ces phénomènes de variations minoritaires restent-ils externes au standard ? dans quelle mesure peuventils au contraire s'y intégrer et le modifier? Les travaux actuels sur la formation d'une "langue littéraire" (cf. G. Philippe), qui prennent en compte l'influence de la norme, de ses représentations, sur ce que l'on pourrait interpréter comme le "style collectif" d'un ensemble d'auteurs, sont en ce sens d'un grand intérêt pour l'évaluation de cette interaction. Il semble toutefois que la norme, si elle joue sans doute un rôle, dont il reste à déterminer l'importance, dans le changement, ne peut s'exercer qu'en sélectionnant, en valorisant ou en éliminant, des variantes déjà en présence. On peut faire l'hypothèse que ces variantes sont le résultat de l'évolution interne du système, et qu'une périodisation prenant pour base des propriétés linguistiques conserve sa pertinence.

En ce qui concerne les changements linguistiques et la reconnaissance de périodes successives, deux démarches, reposant sur des hypothèses théoriques, permettent de dépasser l'empirisme du 'singulier' :

Tout d'abord, la question qui se pose est en fait celle du "noyau stable" et des "marges" : faut-il prendre comme caractéristique définitoire d'une période l'ensemble des points qui n'offrent quasiment aucune possibilité de variation ? Cette position soulève immédiatement un problème qui n'est pas sans importance : une forme, une structure, peuvent être attestées - sans changement particulier - depuis les périodes les plus anciennes, mais ne pas entrer dans les mêmes systèmes d'opposition (les constructions clivées, les interrogatives en est-ce que, qui apparaissent dès l'ancien français, n'ont sans doute pas, à cette époque, le statut central qu'elles possèdent aujourd'hui). Ce sont les relations plus que les éléments euxmêmes qu'il convient de prendre en compte. Cette démarche rencontre par ailleurs un autre obstacle : s'il est indéniable que la rémanence de certaines constructions dans une langue donnée sur une longue durée peut contribuer à définir une 'périodisation' au sein de l'histoire de cette langue, cette attitude conduit vite à devoir superposer ou croiser des 'périodes'.

Un autre angle d'attaque envisageable consiste à centrer l'analyse non sur le permanent, mais sur ce qui change, et à prendre en compte certaines grandes tendances de l'évolution, du mouvement général qui se 
réalise à des vitesses diverses selon les points d'application, et d'en observer les conséquences concrètes et particulières dans les divers sous-systèmes pour essayer de déterminer les moments où se manifeste une rupture ou un aboutissement. Dans le domaine morphosyntaxique, par exemple, trois facteurs au moins seraient ainsi à évaluer :

- la constitution de syntagmes hiérarchisés (avec des relations de dépendance entre les spécifieurs, la tête et les diverses expansions), question en rapport avec celle de l'ordre des éléments, avec l'évolution du schéma V2 (il conviendrait de prendre par exemple en compte des faits comme la fixation de l'objet nominal à la droite du verbe, l'élimination de la séquence VSO par VOS, mais également l'évolution de l'expression du sujet et de celle, parallèle, des déterminants du nom);

- la spécialisation progressive de certaines catégories morphosyntaxiques (mouvement qui peut d'ailleurs être considéré comme une "conséquence" de la tendance à la hiérarchisation), avec, en particulier, la distinction déterminants / pronoms, qui ne s'opère pas au même rythme pour les possessifs, les démonstratifs, ou les indéfinis, mais aussi avec la distinction adverbe / préposition, ou encore, pour prendre un domaine plus restreint, dans les expressions comparatives, la régularisation progressive des formes comme : aussi / si, tant / autant en corrélation avec que ou avec comme.

- l'évolution d'un état de langue proche, sur bon nombre de points, du type synthétique que représente le latin vers un type analytique, facteur qui rejoint en partie les deux précédents. C'est sans doute là la caractéristique la plus "diffuse", la plus transversale, celle qui se prête le moins à des délimitations bien homogènes. On peut en effet considérer qu'elle s'exerce, à des degrés divers sans doute, durant toutes les périodes. La plupart des domaines de la morphosyntaxe se trouvent ici concernés, du système de la négation aux comparatifs, en passant par les formes verbales composées, les formes interrogatives en estce que, les déterminants complexes (beaucoup de), les locutions verbales, les locutions conjonctives ou les locutions prépositionnelles. Dans la mesure où cette tendance est à la fois trop générale et trop "étalée" dans le temps pour permettre de servir de base à un découpage en périodes, c'est sûrement en prenant en compte chacun des sous-domaines de ce type que l'on pourra mettre à l'épreuve ce facteur, qui pourrait compléter alors de façon pertinente les résultats obtenus avec l'analyse des deux tendances précédemment citées.

Cette démarche, on le voit, nécessite d'opérer une double distinction :

- Distinguer des changements de différente nature, et à divers niveaux. Ainsi, des changements singuliers tels que le développement de l'expression du sujet du verbe, le développement de l'emploi des déterminants et la stabilisation (durable) d'un paradigme de spécifieurs, répondraient, au niveau de la morphosyntaxe, à une restructuration du système (la notion de 'paramètre' introduite en GU correspond en partie - en partie seulement - à cette conception).

- Reconnaître, au sein de l'ensemble des changements que connaît une langue, l'existence de 'changements liés', c'est à dire de changements qui, bien que singuliers et distincts les uns des autres, ont en commun de contribuer à un même mouvement de réorganisation du système.

La périodisation pourrait ainsi s'appuyer sur la détermination de moments où convergent différentes réalisations des tendances générales du changement, convergence qui doit être assez importante (quantitativement et qualitativement) pour que l'on puisse parler d'un état de langue d'un type nouveau. 